\title{
The role of percutaneous embolization techniques in the management of dural sinus malformations with atypical angioarchitecture in neonates: report of 2 cases
}

\author{
Bharathi D. Jagadeesan, MD, ${ }^{1,2}$ Andrew W. Grande, MD, ${ }^{1-3}$ Daniel J. Guillaume, MD, ${ }^{2}$ \\ David R. Nascene, MD, ${ }^{1}$ and Ramachandra P. Tummala, MD ${ }^{1-3}$ \\ 1Department of Radiology, ${ }^{2}$ Department of Neurosurgery, and ${ }^{3}$ Department of Neurology, University of Minnesota, \\ Minneapolis, Minnesota
}

\begin{abstract}
Dural sinus malformations (DSMs) are rare congenital malformations that can be midline or lateral in location. Midline DSMs have been reported to have a worse prognosis than lateral DSMs and have traditionally been more difficult to manage. The authors report 2 unusual manifestations of midline DSMs and their management with percutaneous transfontanelle embolization. The first patient (Case 1) presented at 21 days of life with a large midline DSM and multiple highflow dural and pial arteriovenous shunts. The child developed congestive cardiac failure and venous congestion with intracranial hemorrhage and seizures within a few weeks. The second patient (Case 2) presented with a large midline DSM found on prenatal imaging that was determined to be a purely venous malformation on postnatal evaluation. This large malformation resulted in consumptive coagulopathy and apneic episodes from brainstem compression. The patient in Case 1 was treated initially with endovascular embolization and eventually with curative percutaneous-transfontanelle embolization. The patient in Case 2 was treated with percutaneous transfontanelle embolization in combination with posterior fossa decompression and cranial expansion surgery.
\end{abstract}

http://thejns.org/doi/abs/10.3171/2014.12.PEDS145

KEY WORDS arteriovenous fistula; pediatric arteriovenous malformation; dural sinus malformation; percutaneous embolization; vascular disorders

$\mathrm{M}$ IDLINE dural sinus malformations (DSMs) with giant venous lakes are rare vascular malformations that occur in neonates and infants. ${ }^{3,5}$ In this paper we report on a combined percutaneous and endovascular embolization approach. This approach was used in the management of 2 of these malformations with atypical angioarchitectural features, which were not amenable to conservative management or endovascular embolization alone.

\section{Case Reports}

Case 1

History and Examination

A 15-day-old baby girl had a persistent scalp mass, which on ultrasound was shown to be a midline highflow vascular lesion beneath the posterior fontanelle. An MRI study of this lesion demonstrated a DSM with a large partially thrombosed midline venous pouch (MVP) and marked dilation of the posterior one-third of the superior sagittal sinus (SSS), torcula, straight sinus, and the vein of Galen (Fig. 1). Digital subtraction angiography (DSA) performed on Day 21 confirmed the presence of multiple high-flow fistulous and plexiform arteriovenous (AV) shunts to the DSM. The AV shunts to the SSS and upper two-thirds of the MVP were supplied by the middle meningeal arteries (MMAs). The AV shunts to the torcula and the lower one-third of the MVP were supplied by posterior meningeal branches of the vertebral artery (VA), occipital artery, and an artery of Davidoff and Schechter (Fig. 2). There was no evidence for cortical venous reflux. At this time, the child was normal neurologically, had no evidence of cardiac failure, and weighed only $3.4 \mathrm{~kg}$. Therefore treatment was deferred.

\section{Surgery}

The child returned to the hospital on Day 41 with seizures, further enlargement of the DSM, new enlargement

ABBREVIATIONS $\mathrm{AV}=$ arteriovenous; $\mathrm{CCA}=$ common carotid artery; $\mathrm{DSA}=$ digital subtraction angiography; $\mathrm{DSM}=$ dural sinus malformation; $\mathrm{MMA}=$ middle meningeal artery; MVP = midline venous pouch; NBCA = N-butyl cyanoacrylate; $S S S=$ superior sagittal sinus; $V A=$ vertebral artery.

SUBMITTED January 3, 2014. ACCEPTED December 31, 2014.

INCLUDE WHEN CITING Published online April 24, 2015; DOI: 10.3171/2014.12.PEDS145.

DISCLOSURE Dr. Jagadeesan is a consultant for Covidien and MicroVention, and Dr. Grande is in the speaker's bureau for Covidien and Integra. 


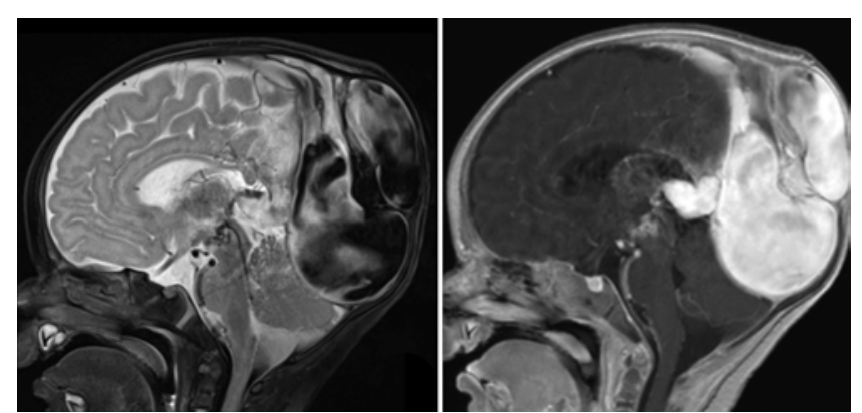

FIG. 1. Case 1. Sagittal T2-weighted (left) and postcontrast T1-weighted (right) images showing a large vascular mass.

of the internal cerebral veins, cortical venous reflux, and small hemorrhages in the right putamen and thalamus. She had also developed early signs of high-output cardiac failure. The patient was discharged home following transarterial embolization of the DSM via MMA feeders on Days 42 and 48, performed by using both ethylene vinyl alcohol copolymer (Onyx 34, Covidien) and $N$-butyl cyanoacrylate (NBCA) (Trufill, Cordis Endovascular), with an approximately $35 \%$ reduction in AV shunting. She returned on Day 62 with seizures, increased deep venous congestion, and small periventricular infarcts. Additional transarterial embolization was performed with Onyx, resulting in another $20 \%$ reduction in flow. This time she was discharged home in anticipation of eventual spontaneous thrombosis of the remainder of the lesion.

The child returned on Day 79 with status epilepticus, a new left thalamic hemorrhage with intraventricular extension, and ventriculomegaly with worsened deep venous congestion. At this time, we determined that the DSM was unlikely to undergo spontaneous thrombosis and that transarterial endovascular embolization with Onyx alone was not likely to be curative as long as the MVP was patent and connected to the sinuses. This was due to the fact that during prior transarterial embolizations the Onyx had a tendency to prematurely penetrate into the MVP rather than the nidus of the DSM. This could have led to eventual penetration of Onyx into the SSS and torcula, with thrombosis of these structures. Transvenous embolization was also not attempted due to concerns about occlusion of the SSS and torcula. Occlusion of these sinuses is undesirable prior to onset of cavernous capture at 6 months of age. There were also limitations to transarterial and transvenous embolization imposed by concerns regarding radiation dose, contrast load, and neurotoxicity from dimethyl sulfoxide.

We therefore decided on a combined percutaneous and endovascular approach to achieve complete elimination of the DSM. On Day 80, we percutaneously punctured the MVP under fluoroscopic guidance and placed a 4-Fr vascular sheath into the MVP. Injection of the sheath showed a $1.6-\mathrm{cm}$-wide midline venous channel between the MVP and SSS, with retrograde filling of the SSS and eventual contrast reflux into the superficial cortical veins. This channel was first obliterated using gel-coated coils deposited through an Echelon 10 microcatheter that was advanced through the sheath, and the upper two-thirds of the MVP was then filled with Onyx, which was also seen to flow retrograde from the MVP into the numerous arterial feeding vessels (Fig. 3). This resulted in obliteration of the upper two-thirds of the MVP, reestablishment of normal drainage in the SSS, and elimination of cortical venous reflux. On Day 81, using a transarterial approach, we embolized the remaining large sections of the DSM nidus by using Onyx and NBCA. On Day 82, by percutaneously placing a micropuncture sheath in the lower one-third of the MVP and injecting Onyx 34 through an Echelon 10 microcatheter into this region, we obliterated the rest of the MVP and its feeders (Fig. 4). Final diagnostic angiography with injections of the common carotid artery (CCA) and VA showed complete obliteration of the DSM.

\section{Outcome}

At 24-month follow-up, the child was neurologically normal and was meeting appropriate developmental milestones. Follow-up MRI showed significant reduction in the thrombosed MVP and no new AV shunts, and the SSS remained patent (Fig. 5). There was no evidence of nephropathy or any short-term radiation injury.

\section{Case 2}

History and Examination

Prenatal ultrasound screening during the second trimester in a G1P0 mother revealed a female fetus with a large midline posterior intracranial cystic lesion. A subse-
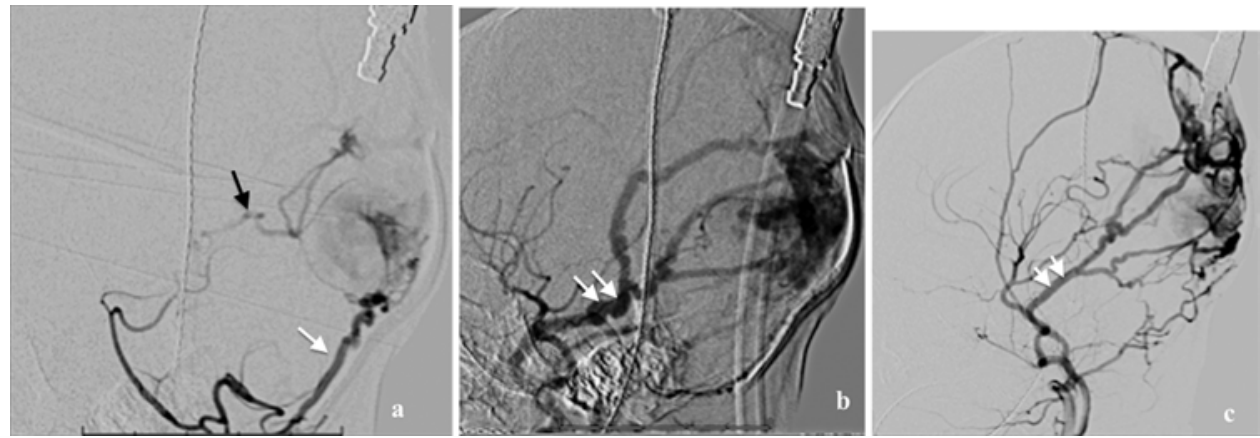

FIG. 2. Case 1. Images from DSA study obtained the day after the initial MRI session. Lateral projections obtained after left VA (a), right CCA (b), and left CCA (c) injections show multiple high-flow AV shunts to the DSM and MVP fed by the posterior meningeal artery (single white arrow) and MMA (double white arrows) as well as an artery of Davidoff and Schecter (single black arrow). 

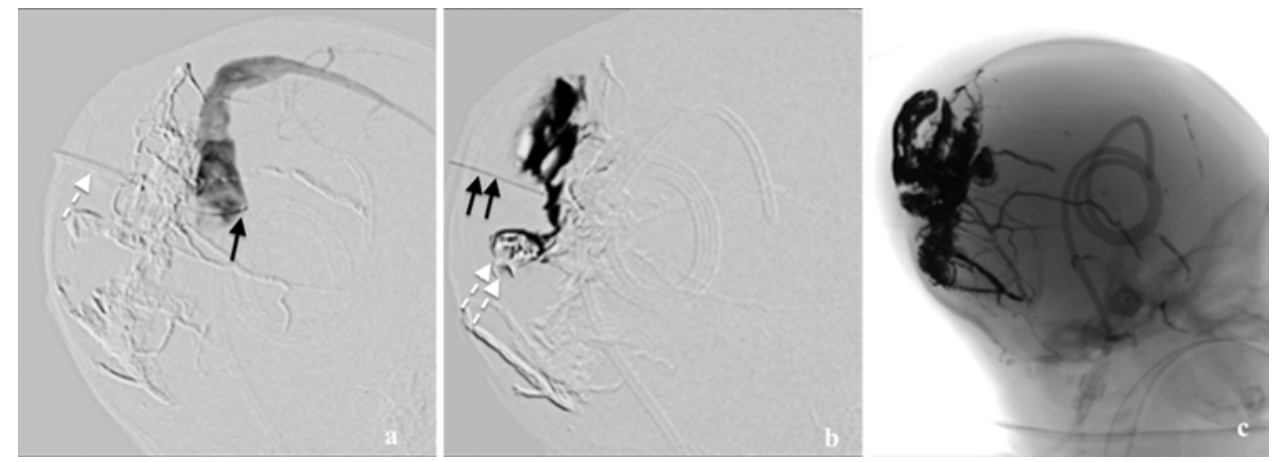

FIG. 3. a: Lateral projection DSA image. Retrograde flow into the SSS after injection of an arterial sheath placed into the MVP (white dashed arrow); note that the tip of the Echelon 10 microcatheter (black arrow) has been advanced from the MVP to the SSS. b: A DSA image in the lateral projection. Coils have been placed in the channel from the MVP to the SSS (double dashed white arrows). The MVP has been selected with a needle (double black arrows), and contrast injection into the MVP prior to Onyx injection shows no opacification of the SSS. c: Final Onyx cast within the superior one-third of MVP.
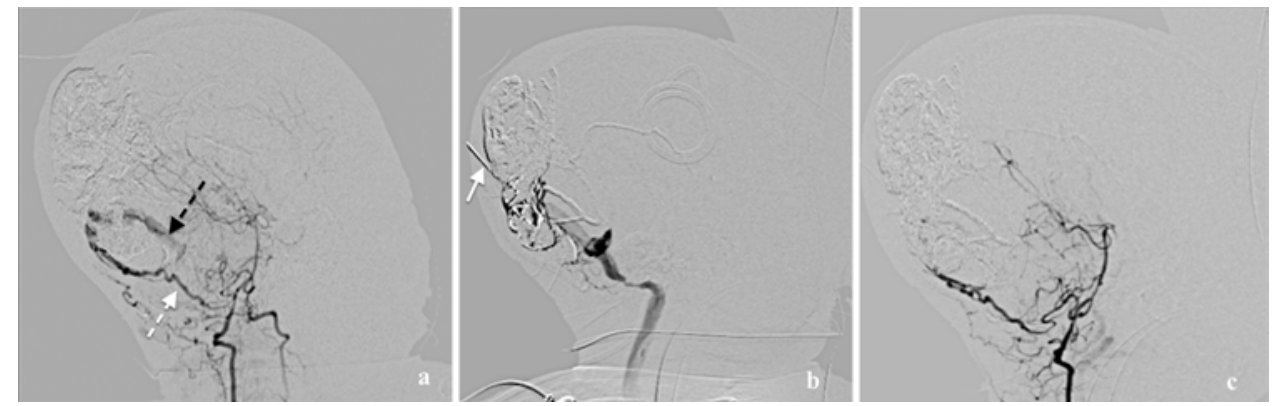

FIG. 4. a: Oblique DSA image after injection of the left VA showing persistent AV shunting into the lower part of the MVP (dashed black arrow) from a posterior meningeal feeder (dashed white arrow). b: Oblique DSA image after contrast injection through a needle placed (solid white arrow) in the lower part of the MVP showing opacification of the internal jugular vein; Onyx was injected at this site. c: Oblique DSA image after injection of the left VA at the end of Onyx injection shows no evidence for residual AV shunting.

quent prenatal uterine MRI study revealed a large midline DSM with thrombus in the expected location of the left transverse sinus, occupying approximately the posterior one-third of the cranial cavity. Serial prenatal MRI studies showed progressive enlargement of this DSM. Following delivery by cesarean section at 35 weeks gestational age, the child underwent an MRI and MR venography session,
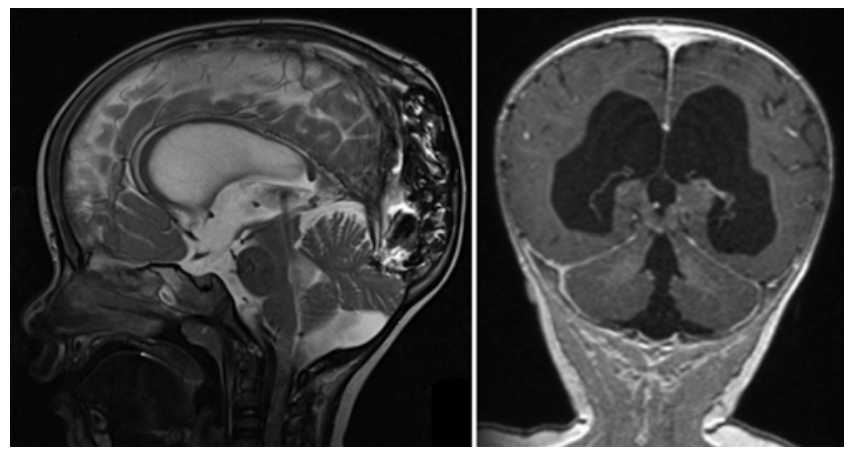

FIG. 5. Case 1. Left: Sagittal T2-weighted image obtained at 18 months' follow-up showing significant shrinking of the vascular mass, which is filled with Onyx. Right: Coronal postcontrast T1-weighted image obtained at the same time showing a patent SSS. followed by a DSA study performed using umbilical arterial catheterization on Day 1 of life.

On the MRI and MR venography studies, the posterior one-third of the SSS, the torcula, and the transverse sinuses were all included in the DSM and could not be distinctly visualized (Fig. 6). The anterior two-thirds of
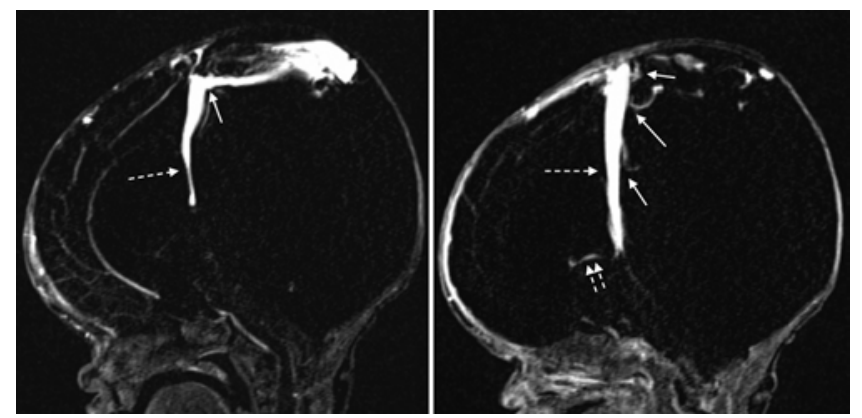

FIG. 6. Case 2. Sagittal slices obtained as part of a post-contrast MR venogram performed on Day 1 after birth showing a venous channel (dashed white arrows) at the anterior margin of the pouch. There are multiple sites of contrast extravasation from the posterior wall of this venous channel into the DSM (white arrows). Note internal cerebral vein draining into this venous channel (double dashed white arrows). 


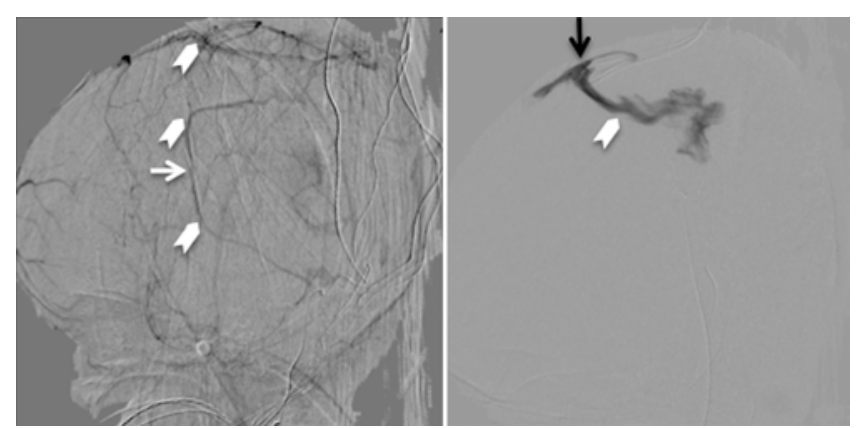

FIG. 7. Left: Lateral DSA projection obtained during the venous phase after right CCA injection: The midline venous channel can be faintly seen as an interrupted line (white arrow). Numerous sites of leakage of contrast from this channel into the DSM are shown (white block arrows). Right: Lateral DSA projection obtained after injection through a needle placed directly into the SSS showing the posterior SSS (black arrow) at its junction with the midline venous channel and shunting into the DSM (white block arrow).

the SSS and the internal cerebral veins were instead seen to drain into a vertical platelike midline venous structure. The posterior wall of this venous channel was apposed to the anterior wall of the DSM. There was rapid flow of blood from the upper end of the posterior wall of this venous channel into the DSM. Numerous less rapid shunts from the posterior wall of the channel into the DSM were also noted more inferiorly. This platelike venous channel was seen to drain inferiorly into the superior petrosal sinuses and eventually the cavernous sinuses. On the DSA study, there was no evidence for AV shunts during the arterial phase, but on the venous phase images from internal and external carotid artery injections, shunting was noted from the posterior wall of a midline venous structure into the DSM at multiple sites, with the most rapid shunting noted at the superior end of this venous structure near the vertex. This finding was confirmed when the SSS was punctured percutaneously with a needle and contrast was injected into the SSS (Fig. 7). The head circumference of the patient at this time was $51 \mathrm{~cm}$. There was no apnea at this time. However, the child suffered from consumptive coagulopathy requiring multiple blood product transfusions.

\section{Surgery}

On Day 2 of life, a micropuncture sheath was percutaneously placed in the SSS via the anterior fontanelle. The superiormost site of shunting from the posterior wall of the vertical venous channel to the DSM pouch was then selected using an Echelon 10 microcatheter, which had been passed through the micropuncture sheath. A total of 4 hydrocoils were then placed at this site to obliterate this communication. We were not successful in selecting the other sites of shunting from the venous channel to the DSM in this way, and therefore we removed the micropuncture sheath. We then used a short 23-gauge butterfly needle to puncture the confluence of the SSS and the vertical venous channel under ultrasound guidance, and then injected this needle to obtain digital roadmaps showing us the sites of communication between the posterior wall of the venous channel and the DSM. We then punctured the DSM venous pouch via a posterior transfontanelle approach by using an 18-gauge spinal needle and then directed the needle anteroinferiorly toward the sites of shunting seen on the roadmap. Localized injections of $30 \%$ NBCA were performed at each of these sites. This was shown to result in a progressive decrease in the extent of shunting from the posterior wall of the vertical venous channel into the venous pouch, as determined by periodic contrast injections through the butterfly needle (Fig. 8).

The child initially did well following this percutaneous procedure, and there was a decrease in head circumference with an increase in thrombus within the DSM on sequential MRIs. However, after 3 weeks the DSM again started enlarging in size and the child experienced apnea due to brainstem compression. For brainstem decompression, a suboccipital decompressive craniectomy and parietooccipital cranial expansion surgery with lateral osteotomies was performed. The child also underwent placement of a ventricular catheter reservoir to allow withdrawal of CSF twice daily for control of intracranial pressure. Although this did result in brainstem decompression, the DSM con-
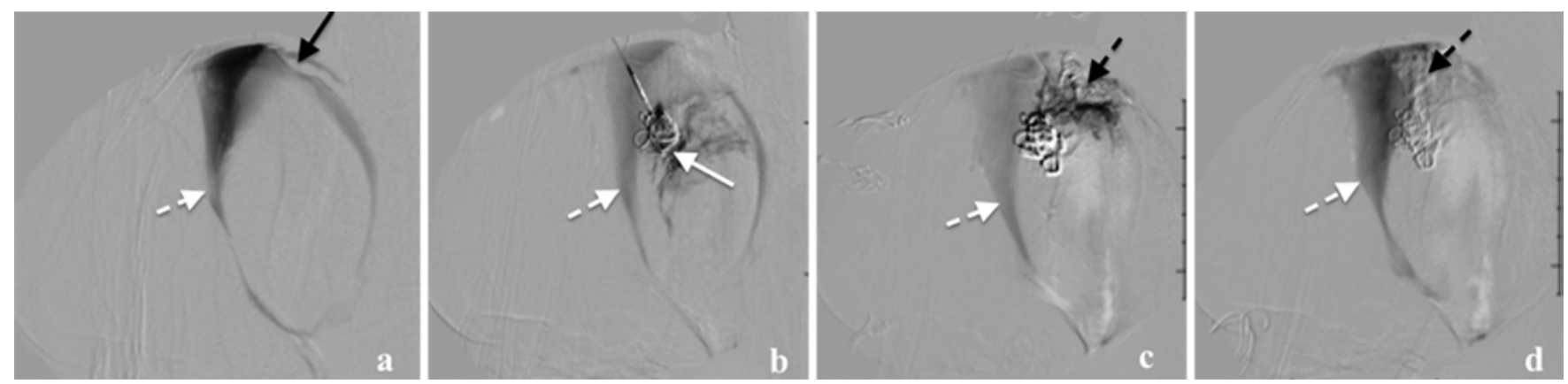

FIG. 8. a: Lateral projection obtained following contrast injection with a butterfly needle, which has been percutaneously positioned at the junction of the SSS and the midline venous channel, showing opacification of the venous channel (dashed white arrows) and prominent leakage of contrast (black arrow) from the posterior wall of the venous channel into the DSM pouch. b: The posteriormost aspect of the SSS has been punctured and coils (white arrow) are being delivered via this access route posterior to the venous channel. c: The DSM pouch has been punctured and an NBCA cast (dashed black arrows) can now be seen partly eliminating the shunt. $d$ : Complete elimination of the shunt, and the NBCA cast is now larger. 


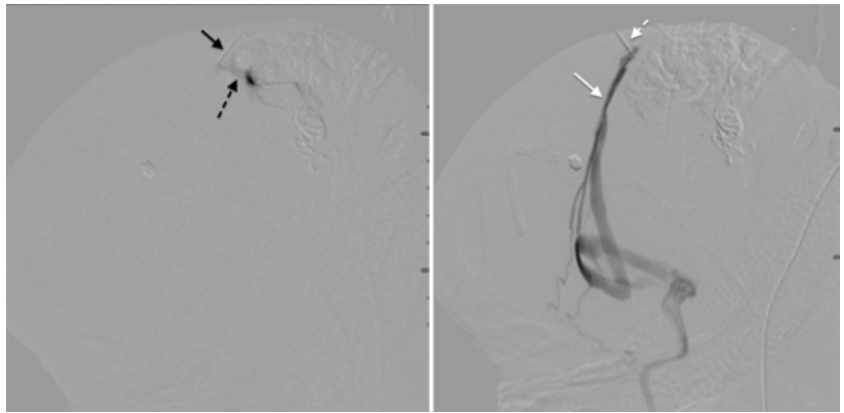

FIG. 9. Left: At 6 weeks after the first embolization, a lateral projection obtained after direct puncture of the DSM pouch with a needle (black arrow) and injection of contrast shows contrast flowing backward into the DSM pouch from residual shunting (dashed black arrow); more NBCA was injected here through this needle. Right: Direct injection (dashed white arrow) of the midline venous channel following completion of NBCA injection shows elimination of shunts and significant remodeling of venous channel (white arrow) when compared with prior images.

tinued to enlarge, prompting suspicion of persistent largevolume shunting from the posterior wall of the venous channel into the DSM. When the child was 6 weeks of age, we again performed a percutaneous injection into the venous channel via a needle placed at the confluence of the SSS and this channel. This showed recurrence of shunting into the DSM from the superiormost part of the posterior wall of the venous channel. Once again, 33\% NBCA was injected at this site after percutaneous puncturing of the DSM as before (Fig. 9). Following this second percutaneous embolization procedure, the DSM showed progressive thrombosis and shrinkage in the following weeks, with significant reduction in pressure on the brainstem and eventual resolution of apnea (Fig. 10). For control of hydrocephalus, a ventriculoperitoneal shunt was placed. The child was eventually discharged home.

\section{Outcome}

At 12-month follow-up, the child continued to do well. Her head circumference had nearly normalized and she continued to achieve more developmental milestones, albeit in a delayed fashion. Repeat MRI showed significant reduction in size of the DSM, with no evidence for con- tinued shunting of blood from the posterior wall of the venous channel into the DSM.

\section{Discussion}

Dural sinus malformations are rare lesions that can be midline or lateral in location. $3,5,6$ When they are midline in location, they have been reported to have a worse prognosis. ${ }^{1}$ Midline DSMs with giant venous pouches are thought to result from uncontrolled dilation of the posterior sinuses, including the torcula and posterior SSS, with associated multiple low-flow AV shunts. ${ }^{9}$ In a young infant with a midline DSM, there is venous hypertension within the sinuses, which is exacerbated when there is also associated immaturity of the jugular veins. Superimposed thrombosis within the venous pouches can then compound impaired cerebral venous drainage and can lead to venous infarction and potentially fatal hemorrhages. ${ }^{3}$ The optimal way to treat these lesions is still unknown given their rarity.

Barbosa et al. have reported their experience with 30 consecutive DSMs with venous lakes. ${ }^{1}$ They managed the lesions in 14 of 30 patients conservatively, and the rest with endovascular embolization. In this series, only 5 of the 14 patients with conservatively managed lesions had a favorable outcome, and cure of the DSM was achieved only in 10 of 30 patients. In contrast, Merzoug et al. described a series of 10 fetuses with DSMs diagnosed on prenatal ultrasound and MRI who received conservative management following live birth. Eight of the 10 neonates in this series had a favorable outcome. ${ }^{6}$ Other authors have also reported on conservative or endovascular management of these lesions, with varying success. ${ }^{2,4,10}$ Successful surgical management of DSMs has also been reported in 2 children, but they did not have associated AV shunts. ${ }^{11}$

Neither of our patients with giant midline DSMs had multiple low-flow dural AV shunts as described by prior authors; on the contrary, one had multiple, very high-flow dural AV shunts, and the other had no AV shunts at all, but rather multiple shunts from a midline venous channel into a giant DSM, causing slow and progressive enlargement of this DSM. Perhaps as a result of these factors, the conservative approach to management did not work well with either infant. The patient in Case 1 became symptomatic, with hemorrhages and seizures, and the patient in Case 2 had a continuous increase in the size of the DSM on pre-
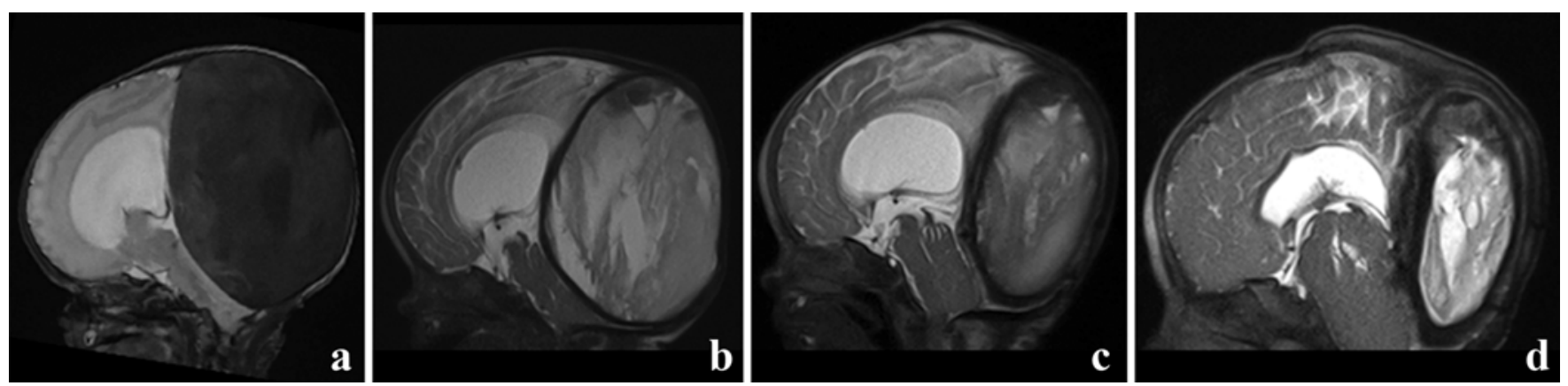

FIG. 10. Case 2. Paramedian sagittal T2-weighted images of the brain obtained at birth (a), 3 months (b), 6 months (c), and 1 year (d) of age showing progressive thrombosis and reduction in volume of the venous pouch as well as progressive reduction in mass effect on brainstem and cerebellum. 
natal and immediate postnatal imaging, with significant pressure on her brainstem. Her DSM did not shrink until her percutaneous embolizations were performed.

The unusual angioarchitecture seen in these patients also required us to deviate from using exclusively transarterial or transvenous approaches for the management of their lesions. We were able to manage the lesions successfully in both patients by partly using a percutaneous approach for embolization. In our experience, this percutaneous method resulted in a much less challenging approach to the location of the shunts draining into the DSM compared with the challenges faced with a transarterial or a modified transvenous approach (obtained by puncturing the SSS in Case 2). A percutaneous transtorcular approach to the management of vein of Galen aneurysmal malformations has been previously described and continues to be used in selected children with this abnormality. ${ }^{7,8}$ However, to the best of our knowledge this simplified method has not been comprehensively described by prior authors for the management of DSMs. Our experience suggests that this approach could perhaps be more widely used in the management of DSMs in infants.

\section{Conclusions}

Midline DSMs with giant venous lakes are rare vascular malformations that occur in neonates and infants. A combined percutaneous and endovascular embolization approach as described here may be useful in the management of some of these malformations, especially those with atypical features that are not amenable to conservative management or purely endovascular embolization.

\section{References}

1. Barbosa M, Mahadevan J, Weon YC, Yoshida Y, Ozanne A, Rodesch G, et al: Dural sinus malformation (DSM) with giant lakes, in neonates and infants. Review of 30 consecutive cases. Interv Neuroradiol 9:407-424, 2003

2. de Haan TR, Padberg RD, Hagebeuk EEO, Aronica E, van Rijn RR, Majoie CBLM, et al: A case of neonatal dural sinus malformation: clinical symptoms, imaging and neuropathological investigations. Eur J Paediatr Neurol 12:41-45, 2008
3. Lasjaunias P, Berenstein A: Dural arteriovenous shunts, in Berenstein A, Lasjaunias P, ter Brugge KG (eds): Surgical Neuroangiography. Berlin: Springer-Verlag, 2004, Vol 2, pp 565-607

4. Liu CA, Chen HC, Luo CB, Guo WY, Mu-Huo Teng M, Chen $\mathrm{HH}$, et al: Dural sinus malformation with arteriovenous fistulae in a newborn: positive outcome following endovascular management. J Chin Med Assoc 75:43-46, 2012

5. McInnes M, Fong K, Grin A, ter Brugge K, Blaser S, Halliday W, et al: Malformations of the fetal dural sinuses. Can J Neurol Sci 36:72-77, 2009

6. Merzoug V, Flunker S, Drissi C, Eurin D, Grangé G, Garel C, et al: Dural sinus malformation (DSM) in fetuses. Diagnostic value of prenatal MRI and follow-up. Eur Radiol 18:692699, 2008

7. Mickle JP, Quisling RG: The transtorcular embolization of vein of Galen aneurysms. J Neurosurg 64:731-735, 1986

8. Mitchell PJ, Rosenfeld JV, Dargaville P, Loughnan P, Ditchfield MR, Frawley G, et al: Endovascular management of vein of Galen aneurysmal malformations presenting in the neonatal period. AJNR Am J Neuroradiol 22:1403-1409, 2001

9. Okudera T, Huang YP, Ohta T, Yokota A, Nakamura Y, Maehara F, et al: Development of posterior fossa dural sinuses, emissary veins, and jugular bulb: morphological and radiologic study. AJNR Am J Neuroradiol 15:1871-1883, 1994

10. Walcott BP, Smith ER, Scott RM, Orbach DB: Dural arteriovenous fistulae in pediatric patients: associated conditions and treatment outcomes. J Neurointerv Surg 5:6-9, 2013

11. Zuccaro G, Argañaraz R, Villasante F, Ceciliano A: Neurosurgical vascular malformations in children under 1 year of age. Childs Nerv Syst 26:1381-1394, 2010

\section{Author Contributions \\ Conception and design: Jagadeesan, Tummala. Acquisition of data: all authors. Analysis and interpretation of data: all authors. Drafting the article: Jagadeesan, Grande. Critically revising the article: all authors. Approved the final version of the manuscript on behalf of all authors: Jagadeesan. Study supervision: Jaga- deesan.}

\section{Correspondence}

Bharathi D. Jagadeesan, Radiology and Neurosurgery, University of Minnesota, 420 Delaware St. SE, Minneapolis, MN 55455. email: jagad002@umn.edu. 